\title{
Nonlinear Resonance Effects During Ion Storage in a Quadrupole Ion Trap
}

\author{
Donald M. Eades, Jodie V. Johnson, and Richard A. Yost \\ Department of Chemistry, University of Florida, Gainesville, Florida, USA
}

Contributions of higher-order fields to the quadrupolar storage field produce nonlinear resonances in the quadrupole ion trap. Storing ions with secular frequencies corresponding to these nonlinear resonances allows absorption of power from the higher-order fields. This results in increased axial and radial amplitudes which can cause ion ejection and collisioninduced dissociation (CID). Experiments employing long storage times and/or high ion populations, such as chemical ionization, ion-molecule reaction studies, and resonance excitation CID, can be particularly susceptible to nonlinear resonance effects. The effects of higher-order fields on stored ions are presented and the influence of instrumental parameters such as radiofrequency and direct current voltage $\left(\mathrm{q}_{\mathrm{x}}\right.$ and $\mathrm{a}_{\mathrm{z}}$ values), ion population, and storage time are discussed. (J Am Soc Mass Spectrom 1993, 4, 917-929)

0 ince the introduction of the quadrupole ion trap detector (ITD) [1] for gas chromatography, a limited number of sophisticated, research-oriented ion trap mass spectrometers (ITMS) have been offered by Finnigan MAT (San Jose, CA). This instrument allows mass-selective isolation, MS $^{n}$ capabilities, and user-defined scanning strategies $[2,3]$. Research with this instrument is the reason for the remarkable transformation of the quadrupole ion trap from a simple benchtop gas chromatograph/mass spcctrometer to potentially one of the most versatile, high performance mass spectrometers.

Pushing the performance of the ion trap beyond that afforded by its original operational mode has led to observations of peculiar behavior, some of which can be attributed to higher-order field effects [4-16]. The quadrupolar field applied to the ion trap can essentially be described by two harmonic oscillators, one in the $z$-direction and one in the r-direction, the strength of which increases linearly with distance from the center of the ion trap. Contributions from higherorder fields distort this relationship between the field strength and displacement from the center leading to "nonlinear" resonances. When the applied quadrupolar field is such that ion secular frequencies (in $\mathrm{z}$ direction or r-direction) correspond to those of higherorder nonlinear resonances, trapped ions may absorb power, resulting in increased axial and radial amplitudes. The specific frequencies at which this phenomenon occurs can be represented as nonlinear resonance lines on the Mathieu stability diagram.

Address reprint requests to Dr. Richard Yost, Department of Chemistry, University of Florida, Gainesville, FL 32611
All quadrupole mass spectrometers possess, to some degree, field distortions resulting in contributions of higher-order fields to the quadrupolar trapping field. In the early 1960s, von Busch and Paul [17] and Dawson and Whetten [18-20] first reported effects from field imperfections in linear quadrupoles and quadrupole ion traps. In general, imperfections in the storage field can be introduced by errors in electrode shape and electrode spacing [18].

Since the initial work by the early pioneers [17-20], relatively few reports describing nonlinear resonance effects in the quadrupole ion trap have appeared in the literature. Recently, however, several publications lave described anomalous behavior due to nonlinear resonances. The first such indications of nonlinear resonance effects were observed during the development and characterization of ion injection systems [4-7] on the ITMS. Louris et al. [5] first showed the dependence of injected ion intensity on the radiofrequency (RF) level. Pedder et al. [6] reported ion intensity from off-axis ion formation and subsequent injection also to be dependent on RF levels, with a strong increase in trapping efficiency near RF levels corresponding to the octopolar resonance at $\boldsymbol{\beta}_{\mathrm{z}}=1 / 2$. Williams et al. [7] reported nonlinear resonance effects during ion injection and showed strong increases in trapping efficiency near RF levels corresponding to $\beta_{z}$ values of $1 / 3$ (dodecapolar) and $1 / 2$ (octopolar). It should be noted that all of the above reports were observed along the $a_{z}=0(0 \mathrm{~V} \mathrm{DC})$ line.

Nonlinear resonance effects have also been observed during resonance excitation collision-induced dissociation (CID) [8-11]. Higher-order field effects during CID experiments, termed "black holes" [8] or "black canyons" [10], resulted in reduced product ion 
signals at RF levels corresponding to $\beta_{z}=1 / 2$ (octopolar) and $\beta_{z}=2 / 3$ (hexapolar). Normal electron ionization (EI) spectra did not show ion intensity losses when the ions were stored for the same amount of time at similar RF levels.

Louris et al. [11] recently described the geometry of all ion traps delivered by Finnigan MAT (and by Perkin Elmer and Varian) as having a "stretched" design. Interestingly, hexapolar effects at $\beta_{z}=2 / 3$ during CID experiments were reported to be reduced with the "stretched" ion trap geometry [11] as compared to traps with theoretical dimensions. This effect was believed to be due to reduced field penetration through the holes of the endcaps of the "stretched" geometry. Field penetration effects were also thought to cause anomalous peaks in spectra while employing smaller-sized ion trap electrodes with normal-sized holes [12]. Prematurely ejected ions were reported to exhibit increasing intensity with decreasing electrode size, but constant hole size. The presence of the anomalous peaks in spectra of high mass-injected ions was attributed to increased field distortion due to the disproportionately large holes in the endcaps; inspection of published spectra [12] suggests that ion ejection was occurring at the hexapolar nonlinear resonance (i.e., at $\beta_{\mathrm{z}}=2 / 3$ instead of $\left.\beta_{z}-1\right)$ causing these peaks to appear at $87 \%$ (i.e., $0.79 / 0.906$ ) of the correct mass-tocharge ratio value. Cameron and Hemberger [13] have rcportcd the appearance of anomalous peaks in EI mass spectra of low mass-to-charge ratio ions while employing a commercially available ion trap. Inspection of the mass-to-charge ratios indicate that the anomalous peaks [13] appear at $87 \%$ of their correct mass-to-charge ratio value, suggesting ion ejection at $\beta_{z}=2 / 3$. Whien employing alternative scanning methods Todd et al. [14] observed ion ejection due to the octopolar nonlinear resonance line at $\beta_{z}=1 / 2$. They observed these effects during "reverse" scans in which the RF and direct current (DC) voltages were reduced (keeping the ratio constant), forcing the scan line to intersect the $\beta_{z}=0$ boundary [14]. Ion ejection at $\beta_{z}=$ $1 / 2$ was also observed during "down" scans in which the $D C$ voltage was increased at constant $R F$ voltage causing the scan line to intersect the $\beta_{z}=0$ boundary [14].

Before presenting and discussing the experimental results, this article will briefly review the theoretical background of nonlinear resonances and offer explanations as to the origin of higher-order fields in the commercial quadrupole ion traps. The experimental observations presented here focus on nonlinear resonance effects observed for stored ions that have not been displaced (i.e., by resonant excitation or ion injection) from the center of the ion trap. These effects have been reproduced with four different mass analyzers on two ITMS systems as well as on a commercial benchtop instrument. Relevant parameters influencing the ability to observe nonlinear resonance effects will also be presented.

\section{Theoretical Treatment}

Early treatment of quadrupole ion trap theory by Dawson and Whetten $[18,20]$ identified areas in the Mathieu stability region where nonlinear resonances could be expected. They correlated experimentally observed peak splitting to theoretically calculated peak shapes and predicted hexapolar and octopolar nonlinear resonance effects due to field imperfections. These predictions came from understanding the applied field potential as well as the contributions of higher-order fields to the trapping field.

The operational mode of most commercial ion traps (Mode II of ref 21) employs grounded endcap electrodes and a combination of DC and RF potentials applied to the ring electrode. The potential at the center of the trap $\left(\Phi_{n}\right)$ becomes [21]:

$$
\Phi_{0}=\frac{U+V \cos \Omega t}{2}
$$

where $U$ is the $D C$ voltage, $V$ is the $R F$ peak voltage, $\Omega$ is the RF drive frequency, and $t$ is time. The potential distribution within an ion trap employing an ideal quadrupolar trapping field, $\Phi_{2}$, can be expressed by the following equation [21]:

$$
\Phi_{2}=\Phi_{0}+\frac{\Phi_{0}}{r_{0}^{2}}\left(r^{2}-2 z^{2}\right)
$$

where $r_{0}$ is the radius of the ring electrode, $r$ represents the cylindrical coordinate along the $r$ axis (centerto-ring distance), and $z$ represents the cylindrical coordinate along the $\mathrm{z}$ axis (center-to-endcap distance).

If contributions of all fields (dipolar, quadrupolar, and higher-order fields) are considered, a power-series can be written to describe the field potential about the point $(z=0, r=0)$ as follows [22]:

$$
\begin{aligned}
& \Phi_{\text {Total }} \\
& \qquad=\Phi_{0}+\Phi_{0}\left(A_{1} \Phi_{1}+A_{2} \Phi_{2}+A_{3} \Phi_{3}+A_{4} \Phi_{4}+\cdots\right)
\end{aligned}
$$

where the subscripts represent the field order (i.e., dipolar $=1$, quadrupolar $=2$, hexapolar $=3$, etc.) and $A_{N}$ are weighting factors dependent on the relative contribution of each field. Note that the $\Phi_{0}$ term has been factored out. The equation for the dipolar field [22] is

$$
\Phi_{1}=\frac{z}{r_{0}}
$$


and the equations for the higher-order fields are [22]:

$$
\begin{gathered}
\Phi_{3}=\frac{\left(2 z^{3}-3 z r^{2}\right)}{r_{0}^{3}} \\
\Phi_{4}=\frac{\left(8 z^{4}-24 z^{2} r^{2}+3 z^{4}\right)}{r_{0}^{4}} \\
\Phi_{5}=\frac{\left(8 z^{5}-40 z^{3} r^{2}+15 z r^{4}\right)}{r_{0}^{5}} \\
\Phi_{6}=\frac{\left(16 z^{6}-120 z^{4} r^{2}+90 z^{2} r^{4}-5 r^{6}\right)}{r_{0}^{6}}
\end{gathered}
$$

where the $\Phi_{N}$ functions (spherical harmonics) have numerical coefficients chosen to satisfy the Laplace equation. No contribution from higher-order terms (i.e., $\mathrm{A}_{\mathrm{N}>2}=0$ ) and no application of a dipolar field yields the ideal quadrupolar field equation expressed in eq 2. Construction of theoretically correct electrodes yields zero contribution from higher-order even terms (i.e., octopolar, dodecapolar). Higher-order odd terms (i.e., hexapolar, decapolar) can be eliminated by making both the electrode surfaces and the applied potentials with reflection symmetry in the $\mathrm{z}=0$ plane [22]. Although the relationship between $r_{0}$ and $z_{0}$ (center-toendcap distance) for a pure quadrupolar field is typically $r_{0}^{2}=2 z_{0}^{2}[20]$, other dimensions can be used provided the electrodes conform to equipotential surfaces of a pure quadrupolar field according to the equations:

$$
\begin{gathered}
\frac{1}{r_{0}^{2}}\left(r^{2}-2 z^{2}\right)=1 \\
\frac{1}{2 z_{0}^{2}}\left(r^{2}-2 z^{2}\right)=-1
\end{gathered}
$$

for the ring and endcap electrodes, respectively [23].

Although the amount of contribution of each higher-order field is difficult to determine, the locations of nonlinear resonances within the stability region can be predicted. In general, higher-order even (e.g., octopolar, dodecapolar) nonlinear resonances can be predicted with solutions for the harmonic oscillator as described by the following equation [20]:

$$
\frac{1}{2} K \beta_{r}+\frac{1}{2}(N-K) \beta_{z}=1
$$

where $\mathrm{N}$ is the field order and $\mathrm{K}$ can have the values $\mathrm{N}, \mathrm{N}-2, \mathrm{~N}-4$, etc. The parameters $\beta_{\mathrm{z}}$ and $\beta_{\mathrm{r}}$ yield values between 0 and 1 and are related to the ion's secular frequencies in the radial $\left(\omega_{\mathrm{r}}\right)$ and axial $\left(\omega_{\mathrm{z}}\right)$ directions, with $\beta_{\mathrm{r}}=2 \omega_{\mathrm{r}} / \Omega$ and $\beta_{\mathrm{z}}=2 \omega_{\mathrm{z}} / \Omega$. Higher-order even fields are introduced by axially symmetric field distortions which are caused by any deviation from the theoretical dimension in which the departures from ideal are equal on either side of the $z=0$ plane. For example, this type of error can be caused by equal deviations in each endcap-to-ring distance, as in the "stretched" ion trap [11].

Higher-order odd nonlinear resonances (e.g., hexapolar, decapolar) are described by the following equation [24]:

$$
\frac{1}{2}(\mathrm{~N}-\mathrm{K}) \beta_{\mathrm{r}}+\frac{1}{2} \mathrm{~K} \beta_{\mathrm{z}}=1
$$

where $\mathrm{N}, \mathrm{K}, \boldsymbol{\beta}_{\mathrm{r}}$, and $\boldsymbol{\beta}_{\mathrm{z}}$ have the same definitions as given above. Higher-order odd fields are introduced by axially asymmetric field distortions which are caused by any deviation from the theoretical dimension in which the departures from ideal are different on each side of the $z=0$ plane. For example, this type of error can be caused by employing two endcaps differing in endcap-to-ring distances, geometries, or potentials.

Table 1 lists the nonlinear resonances possessing only positive terms (i.e., $\mathrm{K} \geq 0$ ) for distortions due to higher-order fields. A thorough theoretical description of all predicted nonlinear resonances has been offered by Wang et al. [24]. Higher-order field distortions observed and discussed in this article are labeled by an asterisk (*). The $\beta_{\mathrm{z}}$-type resonances absorb power in the z-direction, $\beta_{\mathrm{r}}$-type resonances absorb power in the r-direction, sum resonances absorb power in both directions, and difference resonances (not shown in Table 1) exchange power between $r$ and $z$ oscillations [24]. It should be noted that higher-order field contributions (odd or even) will become weaker with increasingly higher order (larger N) [24]; hence the hexapolar field is the most influential of the higher-order odd fields

Table 1. Nonlinear resonances for higher-order fields $(n=3$ to $n=8)$ positive and sum resonances only $(K \geq 0)$

\begin{tabular}{clcccc}
\hline $\begin{array}{c}\text { Third } \\
\text { Order } \\
\text { (Hexapolar) }\end{array}$ & $\begin{array}{c}\text { Fourth } \\
\text { Order } \\
\text { (Octopolar) }\end{array}$ & $\begin{array}{c}\text { Fifth } \\
\text { Order } \\
\text { (Decapolar) }\end{array}$ & $\begin{array}{c}\text { Sixth } \\
\text { Order } \\
\text { (Dodecapolar) }\end{array}$ & $\begin{array}{c}\text { Seventh } \\
\text { Order } \\
\text { (Tetradecapolar) }\end{array}$ & $\begin{array}{c}\text { Eighth } \\
\text { Order } \\
\text { (Hexadecapolar) }\end{array}$ \\
\hline \hline$\beta_{\mathrm{z}}=2 / 3^{\star}$ & $\beta_{\mathrm{z}}=1 / 2^{\star}$ & $\beta_{\mathrm{z}}=2 / 5$ & $\beta_{\mathrm{z}}=1 / 3^{\star}$ & $\beta_{\mathrm{z}}=2 / 7$ & $\beta_{\mathrm{z}}=1 / 4$ \\
$\beta_{\mathrm{z}} / 2+\beta_{\mathrm{r}}=1$ & $\beta_{\mathrm{r}}=1 / 2^{\star}$ & $3 / 2 \beta_{\mathrm{z}}+\beta_{\mathrm{r}}=1$ & $\beta_{\mathrm{r}}=1 / 3$ & $1 / 2 \beta_{\mathrm{z}}+3 \beta_{\mathrm{r}}=1$ & $\beta_{\mathrm{r}}=1 / 4$ \\
& $\beta_{\mathrm{z}}+\beta_{\mathrm{r}}=1^{*}$ & $1 / 2 \beta_{\mathrm{z}}+2 \beta_{\mathrm{r}}=1$ & $\beta_{\mathrm{z}}+2 \beta_{\mathrm{r}}=1$ & $3 / 2 \beta_{\mathrm{z}}+2 \beta_{\mathrm{r}}-1$ & $\beta_{\mathrm{z}}+3 \beta_{\mathrm{r}}-1$ \\
& & & $2 \beta_{\mathrm{z}}+\beta_{\mathrm{r}}=1$ & $5 / 2 \beta_{\mathrm{z}}+\beta_{\mathrm{r}}=1$ & $2 \beta_{\mathrm{z}}+2 \beta_{\mathrm{r}}=1$ \\
& & & & & $3 \beta_{\mathrm{z}}+\beta_{\mathrm{r}}=1$ \\
\hline
\end{tabular}


and the octopolar is the most influential of the higherorder even fields.

Figure 1 is a Mathieu stability diagram for an ion plotted in $\mathrm{q}_{\mathrm{z}}-\mathrm{a}_{\mathrm{z}}$ space along with the nonlinear resonances corresponding to the higher-order fields that significantly contribute to the quadrupolar field. The Mathieu parameters, $a_{x}$ and $q_{z}$, are proportional to the DC and RF voltages as described by the following equations [21]:

$$
\begin{gathered}
q_{z}=-2 q_{r}=\frac{8 e V}{m\left(r_{0}^{2}+2 z_{0}^{2}\right) \Omega^{2}} \\
a_{z}=-2 a_{r}=-\frac{16 e U}{m\left(r_{0}^{2}+2 z_{0}^{2}\right) \Omega^{2}}
\end{gathered}
$$

where $e$ is the charge on the ion and $m$ is the mass of the ion.

The origin of higher-order nonlinear resonances stems from the fact that it is buth impussible and impractical to construct an ideal quadrupole ion trap. Theory predicts hyperbolic electrodes of infinite dimension which, in reality, must be truncated for construction of a reasonably sized ion trap. Truncation of the electrodes results in axially symmetric distortions generating higher-order even nonlinear resonances [22]. Higher-order odd nonlinear resonances are introduced due to the inherent differences between the entrance and exit endcaps [22]. This axially asymmetric distortion may arise from machining flaws or differences in the number or size of holes in each endcap. The exit endcap of Finnigan MAT ion traps has six holes symmetrically positioned around a center hole of equal size located at its apex. These holes are used for ion extraction to the detector. The entrance endcap of

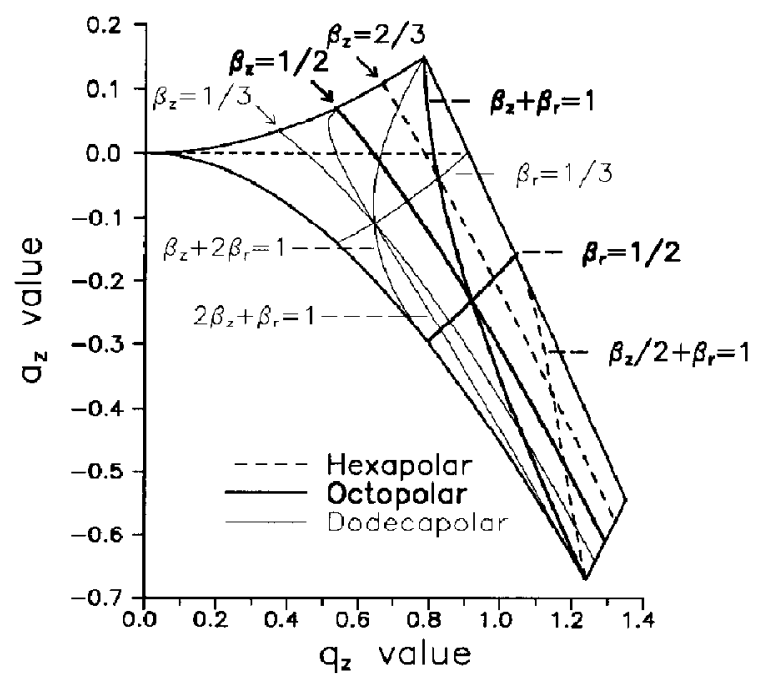

Figure 1. Mathieu stability diagram $\left(q_{z}-a_{z}\right.$ space) showing locations of nonlinear resonances due to hexapolar (third-order), octopolar (fourth-order), and dodecapolar (sixth-order) fields.
Finnigan MAT ion traps has one center hole (the same size as each of the exit holes) at its apex used for introduction of electrons. These holes introduce distortions in the pure quadrupolar field both from gaps in the equipotential electrode surface and from field penetration. With the differences in endcap holes and in the applied potentials behind each endcap (i.e., due to electron gate voltage and dynode or multiplier voltage), field penetrations may differ, resulting in axially asymmetric field distortions. It should be noted that the field penetration due to the dynode and /or multiplier potentials will depend on their distance from the endcap electrode; furthermore, the presence of a grounded shield between the endcap and dynode/ multiplier assembly in some Finnigan MAT ion trap instruments will significantly reduce field penetration. Griffiths et al. [15] investigated field distortions due to holes in the endcaps of a noncommercial ion trap and reported effects from the octopolar resonance at $\beta_{z}+$ $\beta_{\mathrm{r}}=1$. Since the endcaps were identical in geometry and potential (axially symmetric), it follows that a higher-order even nonlinear resonance would be observed.

The endcap electrodes of all ion traps delivered by Finnigan MAT are displaced from the theoretical dislance $\left(\mathrm{z}_{0}\right)$ by approximately $10.7 \%$, with no correction of the electrode surface to conform to pure quadrupolar geometry [11]. The ring electrode radius $\left(r_{0}\right)$ is 1.0 $\mathrm{cm}$ and the hyperbolic endcap electrode surfaces are constructed to correlate to $\mathrm{z}_{0}$ equal to $0.7071 \mathrm{~cm}$ (i.e., $\mathrm{r}_{0}^{2}=2 \mathrm{z}_{0}^{2}$ ); the $10.7 \%$ "stretch" yields a $\mathrm{z}_{0}$ dimension equal to $0.783 \mathrm{~cm}$ [11]. Actual measurements of Finnigan MAT ion trap electrodes have shown $z_{0}$ equal to $0.7811 \mathrm{~cm}$ [25]. According to theory, this geometry will introduce even-order nonlinear resonances (i.e., octopolar, dodecapolar) due to the axially symmetric deviation from the theoretical quadrupolar dimensions. It should be noted that the contribution of higher-order fields to the quadrupolar field is not entirely detrimental. Indeed, Franzen [26] reported significant improvements in trapping efficiency with the introduction of $3 \%$ octopolar field. Also, as discussed in the introduction, Louris et al. [11] have shown improved ion trap performance with the "stretched" design. In particular, they reported an overall improvement in resolution as well as decreased hexapolar "black hole" effects during CID experiments near $\beta_{z}=2 / 3[11]$.

\section{Experimental}

All experiments were performed on two Finnigan MAT ITMS systems, each maintained at $100^{\circ} \mathrm{C}$. Similar experiments were performed with customized software on a Finnigan MAT ITS-40 benchtop instrument [27]. Careful measurements indicated that both ITMS analyzer assemblies were of the same geometry as described by Finnigan MAT [11]. Helium (Liquid Air, Walnut Creek, CA) was used as the buffer gas; isobu- 
tane (Alphagaz, LaPorte, TX), high-performance liquid chromatography grade methanol and acetonitrile (Fisher Scientific, Fair Lawn, NJ), and methane (Matheson Gas Products, Morrow, GA) were introduced via a fine metering valve (Negretti, Southampton, England). The n-alkylbenzenes (Eastman Kodak, Rochester, NY) were introduced via a Granville-Phillips (Boulder, $\mathrm{CO}$ ) variable leak valve mounted directly on a $1 / 2$-inch O.D. stainless steel probe. Pressures were read directly off a Bayard-Alpert ionization gauge controller and are reported with no correction factors. Typical indicated pressures were $1.1 \times 10^{-4}$ torr for helium (corresponding to a corrected pressure of approximately $9 \times 10^{-4}$ torr), $1.0 \times 10^{-5}$ torr for methane and other chemical ionization $(\mathrm{CI})$ reagent gases, and $2.0 \times 10^{-6}$ torr for the n-alkylbenzenes. Carbaryl (Pesticide Chemical Repository, Research Triangle Park, NC) was introduced via a solids probe heated to $100{ }^{\circ} \mathrm{C}$.

Ion simulations were performed with the ITSIM program [28] and HYPERION [29]. All calculations were performed according to equations for the "stretched" ion trap design [30]. Ion trap software used for all experiments was developed in our laboratory (written by Nathan Yates, University of Florida), and allowed data acquisition while incrementing the RF voltage by 1 RF digital-to-analog converter (DAC) step. A FORTH procedure was used to increment the RF voltage for all ion storage studies. Data reduction was then performed using the BASIC program, CHROLIST (written by Randal Pedder and Nathan Yates, University of Florida), which retrieves original ITMS data files, averages selected mass intensities, and writes the data into a tabular ASCII format. The threedimensional (3D) plot and the topographic plot were generated with SURFER (Golden Software, Inc., Golden, $\mathrm{CO}$ ) by creating a $223 \times 223 \mathrm{grid}$ of the data by a spline smoothing algorithm provided by SURFER. The $\mathrm{q}$, values were calculated by first determining the low mass cutoff by multiplying the corresponding DAC value by the experimentally determined slope of the calibration curve for low $m / z$ ions (e.g., 6.314 DACS/amu). The low mass cutoff was then used in the equation below to calculate the $\mathrm{q}_{2}$ value:

$$
\mathbf{q}_{z}=\frac{\text { LowMassCutoff } * 0.906}{m / z}
$$

Scan functions used for these experiments employed a $1 \mathrm{~ms}$ ionization time at $q_{z}=0.3$ followed by mass-selection of the ion of interest by RF/DC isolation at $\mathrm{q}_{z}=0.78, \mathrm{a}_{\mathrm{z}}=0.15$ [31]. The $\mathrm{RF}$ voltage was then ramped to the desired $\mathrm{q}_{z}$ value for ion storage for up to $100 \mathrm{~ms}$. The RF drive frequency was $1.1 \mathrm{MHz}$; axial modulation was employed at $530 \mathrm{kHz}$ (resonant ejection at $\mathrm{q}_{z}=0.906$ ) at $6 \mathrm{~V}_{\mathrm{p}-\mathrm{p}}$ during the mass-selective instability scan. The electron multiplier (EM) voltage was set to yield $10^{5}$ gain and no dynode voltage was used. A grounded shield was present in front of the dynode /EM assembly.

\section{Results and Discussion}

Generally speaking, higher-order field effects can be observed when a relatively high ion population is stored for several milliseconds at a fixed $\left(q_{z}, a_{z}\right)$ point corresponding to a nonlinear resonance condition. During a normal EI scan, ions are typically stored at a fixed $\left(\mathrm{q}_{\mathrm{z}}, \mathrm{a}_{\mathrm{z}}\right)$ point for relatively short times $(<10 \mathrm{~ms})$; thus, higher-order field effects are not typically observed in EI spectra.

Also contributing to the observation of nonlinear resonance effects is ion displacement from the center of the trap. Near the center of the ion trap, higher-order field potentials reduce quickly, yielding a purely quadrupolar field. Higher-order field potentials increase their contribution to the trapping field with increasing distance away from the center of the ion trap. Ions stored in the outer regions of the trap will therefore absorb more power from the higher-order fields. Ion displacement from the center of the trap can be caused by:

1. high ion population (space charge) causing ion-ion repulsions;

2. absence of buffer gas requiring a longer time for ions to cool to the center of the trap;

3. resonance excitation causing increased axial and/or radial motion;

4. injection of ions near the endcap or ring electrodes;

5. ion formation near the endcap or ring electrodes (e.g., laser desorption near ring); and

6. application of $D C$ voltages which alters axial and radial motion.

\section{Nonlinear Resonance Effects During Storage of $\mathrm{Cl}$ Reagent Ions}

Nonlinear resonance effects originally were observed in our laboratory during storage of $\mathrm{CI}$ reagent ions [16]. Investigations into mass-selected $\mathrm{CI}$ with various reagent ions led to the discovery that specific combinations of $R F$ and $D C$ voltages gave unfavorable $C I$ reaction conditions. Operation along the $\mathrm{a}_{\mathrm{z}}=0$ line $(D C=0 \mathrm{~V})$ with variation of the $R F$ voltage (and thereby the $q_{z}$ of the reagent and analyte ions) resulted in analyte ion signal minima near $q_{z}-0.65$ and 0.8 for the $\mathrm{CI}$ reagent ion. This trend was observed for various $\mathrm{CI}$ reagent ions and analytes. Further investigations revealed that the loss in analyte ion signal at these specific $\mathrm{q}_{\mathrm{z}}, \mathrm{a}_{\mathrm{z}}$ values was due to loss of $\mathrm{CI}$ reagent ions. Protonated methanol $(m / z 33)$, protonated acetonitrile $(m / z 42)$, and the $(M-H)^{+}$ion of isobutane $(m / z 57)$ were ejected near $\mathrm{q}_{\mathrm{z}}=0.65\left(\beta_{\mathrm{z}}=\right.$ $1 / 2)$ and $0.8\left(\beta_{z}=2 / 3\right)$, while the $\mathrm{C}_{2} \mathrm{H}_{5}^{+}(m / z 29)$ and $\mathrm{C}_{3} \mathrm{H}_{5}^{+}(\mathrm{m} / z 41)$ ions of methane underwent $\mathrm{CID}$ as well as ejection near these $q_{z}$ values.

An example of this behavior is shown in Figure 2, where mass-selected $\mathrm{C}_{2} \mathrm{H}_{5}^{+}$ions of methane were stored for $50 \mathrm{~ms}$ at various $\mathrm{RF}$ levels (i.e., $\mathrm{q}_{\mathrm{z}}$ values) 


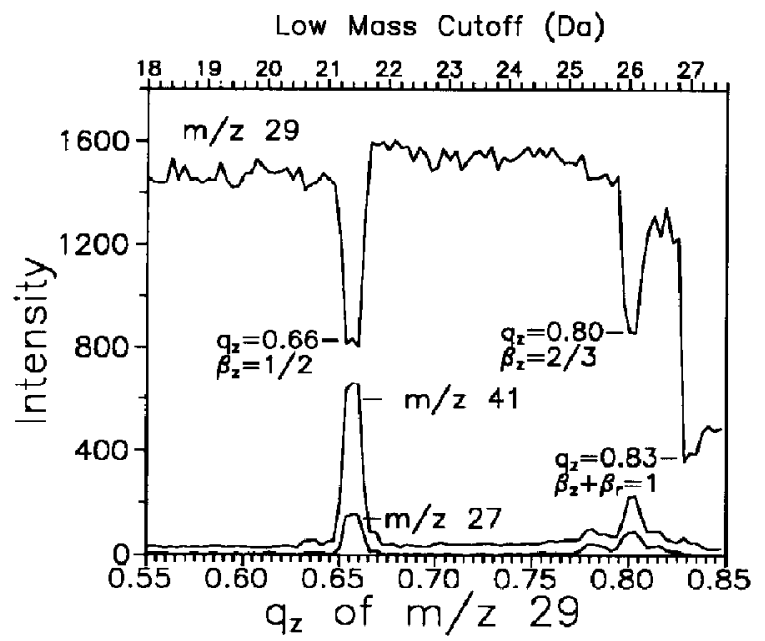

Figure 2. Intensity of $m / z 29\left(\mathrm{C}_{2} \mathbf{H}_{5}^{+}\right)$and $\mathrm{CID}$ product ions $\mathrm{C}_{2} \mathrm{H}_{3}^{+}(m / z 27)$ and $\mathrm{C}_{3} \mathrm{H}_{5}^{1}(m / z 41)$ versus $\mathrm{q}_{2}$ of $m / z 29$ for 50 ms storage $\left(a_{2}=0\right)$ of mass-selected $m / z 29$ ions. Intensity losses correspond to $\beta_{z}=1 / 2$ (octopolar), $\beta_{z}=2 / 3$ (hexapolar), and $\beta_{z}+\beta_{r}=1$ (octopolar).

along the $\mathrm{a}_{z}=0$ line. Near $\mathrm{q}_{z}=0.65, \mathrm{q}_{z}=0.8$, and $\mathrm{q}_{\mathrm{z}}=0.83$ signal minima for $m / z 29$ are observed along with corresponding increases in $m / z 27$ and 41 . The loss of ion signal beyond $\mathrm{q}_{\mathrm{z}}=0.85$ is due to inefficient storage of the reagent ion near the ejection edge $\left(q_{z}=0.906\right)$. The $q_{z}$ values at the signal minima correspond to the $\beta_{z}=1 / 2$ (octopolar), $\beta_{z}=2 / 3$ (hexapolar), and $\beta_{x}+\beta_{\mathrm{r}}=1$ (octopolar) nonlinear resonances. At these $\mathrm{q}_{\mathrm{z}}$ values, the $\mathrm{C}_{2} \mathrm{H}_{5}^{+}$ion of methane absorbs power from the higher-order fields and undergoes CID to form $m / z 27$, which then reacts with neutral methane to form $m / z 41$ as follows [32]:

$$
\begin{gathered}
\mathrm{C}_{2} \mathrm{H}_{5}^{+} \stackrel{\mathrm{CID}}{\longrightarrow} \mathrm{C}_{2} \mathrm{H}_{3}^{+}+\mathrm{H}_{2} \\
m / z 29 \\
m / z 27 \\
\mathrm{C}_{2} \mathrm{H}_{3}^{+}+\mathrm{CH}_{4} \underset{m / z 27}{\mathrm{C}} \mathrm{C}_{3} \mathrm{H}_{5}^{+}+\mathrm{H}_{2} \\
m / \mathrm{z} 41
\end{gathered}
$$

The reaction pathway has been confirmed by resonance excitation CID of $m / z 29$ at other arbitrarily chosen $\mathrm{q}_{\mathrm{z}}$ values. The inefficient storage near $\mathrm{q}_{x}=0.65$ and 0.8 agrees with the $\mathrm{q}_{\mathrm{z}}$ values observed for loss of product ion signal from MS/MS experiments [8-11].

\section{Mapping the Stability Region}

An ideal Mathieu stability region, as defined in Figure 1 , would exhibit uniform storage efficiency throughout the entire stability region and display very sharp edges separating the stable and unstable regions. To map the experimental stability region, the $\mathrm{C}_{2} \mathrm{H}_{5}^{+}$ion of methane was used as a chemical probe. With only methane in the ion trap, the $\mathrm{C}_{2} \mathrm{H}_{5}^{+}$ion $(m / z$ 29) was mass-selected and stored for $100 \mathrm{~ms}$. Although nonlinear resonance effects can be observed without mass-isolation, they are more apparent by simplifying the mass spectrum to ions of a single mass-to-charge ratio. At 32 different $a_{z}$ values (DC voltages), the $q_{z}$ value of the $100 \mathrm{~ms}$ storage period was varied by incrementing the RF voltage, as described in the Experimental section.

Shown in Figure 3a is the experimentally determined stability region for the $\mathrm{C}_{2} \mathrm{H}_{5}^{+}$ion. This 3D map, plotted with $\mathrm{a}_{\mathrm{z}}$ along the $\mathrm{y}$-axis, $\mathrm{q}_{\mathrm{z}}$ along the $\mathrm{x}$-axis, and intensity along the vertical axis, shows that the experimental stability region is far from ideal. Ion losses in the 3D map are observed along iso- $\beta$ lines corresponding to $\beta_{\mathrm{z}}=1 / 3$ (dodecapolar), $\beta_{\mathrm{z}}=1 / 2$ (octopolar), and $\beta_{z}=2 / 3$ (hexapolar). Figure 3b shows the same data in terms of a topographic plot. Note that the data were acquired only from $\mathrm{a}_{\mathrm{z}}=+0.05$ to -0.30 and that the solid line is the theoretically predicted stability boundary. The topographic plot makes the ion losses near the $\beta_{z}=1$ stability edge more apparent. The early onset of ejection near this border arises from the long storage times. Evident in the topographic plot, but less apparent in the 3D plot, are ion losses along the iso- $\beta$ line corresponding to $\boldsymbol{\beta}_{\mathrm{r}}=1 / 2$ (octupolar). Although difficult to observe in these plots, the data suggest contributions from the $\beta_{\mathrm{x}}+2 \beta_{\mathrm{r}}=1$ (dodecapolar) and $\beta_{z}+\beta_{\mathrm{r}}=1$ (octopolar) sum resonance lines. Results from ion injection from an external offaxis ion source also indicate some influence from these sum resonance lines (Pedder, private communication). Note that the most stable portion is the upper left of the stability region (i.e., $q_{z}<0.61$ and $a_{z}>-0.05$ ).

It should be noted that experimentally determined $\mathrm{q}_{\mathrm{x}}$ values (and thus $\beta_{\mathrm{z}}$ values) which correspond to the observed signal minima are slightly higher than the theoretically predicted values for the nonlinear resonances. In other words, the experimentally observed nonlinear resonances fall slightly to the right of the theoretical iso- $\beta_{z}$ lines. There are several factors that may contribute to the observed discrepancy. The most important factor is believed to be the ion population stored in the ion trap during the experiments. As discussed in a following section (Ion Population), observed signal minima shift to higher $q_{x}$ values with increasingly higher ion population. The reason for this is believed to be ion frequency shifts due to the effects of space charge or higher-order fields. Spacecharge-induced shifts of ion resonance and stability boundaries have been addressed by other authors [33-35]; this treatment views space-charge effects as the application of a DC potential on the electrodes. This causes the ions to be stored at an $a_{z}$ value that deviates from theoretical predictions. Wang and Franzen [36] have shown with simulations that shifts in ion secular frequency and shifts in the stability edge can be caused by contributions of higher-order fields. Although no obvious influence on ion calibration was observed, these effects may contribute to errors in calculations of $\mathrm{q}_{z}$ values and, consequently, $\beta_{z}$ values. Another factor contributing to errors in these calcula- 

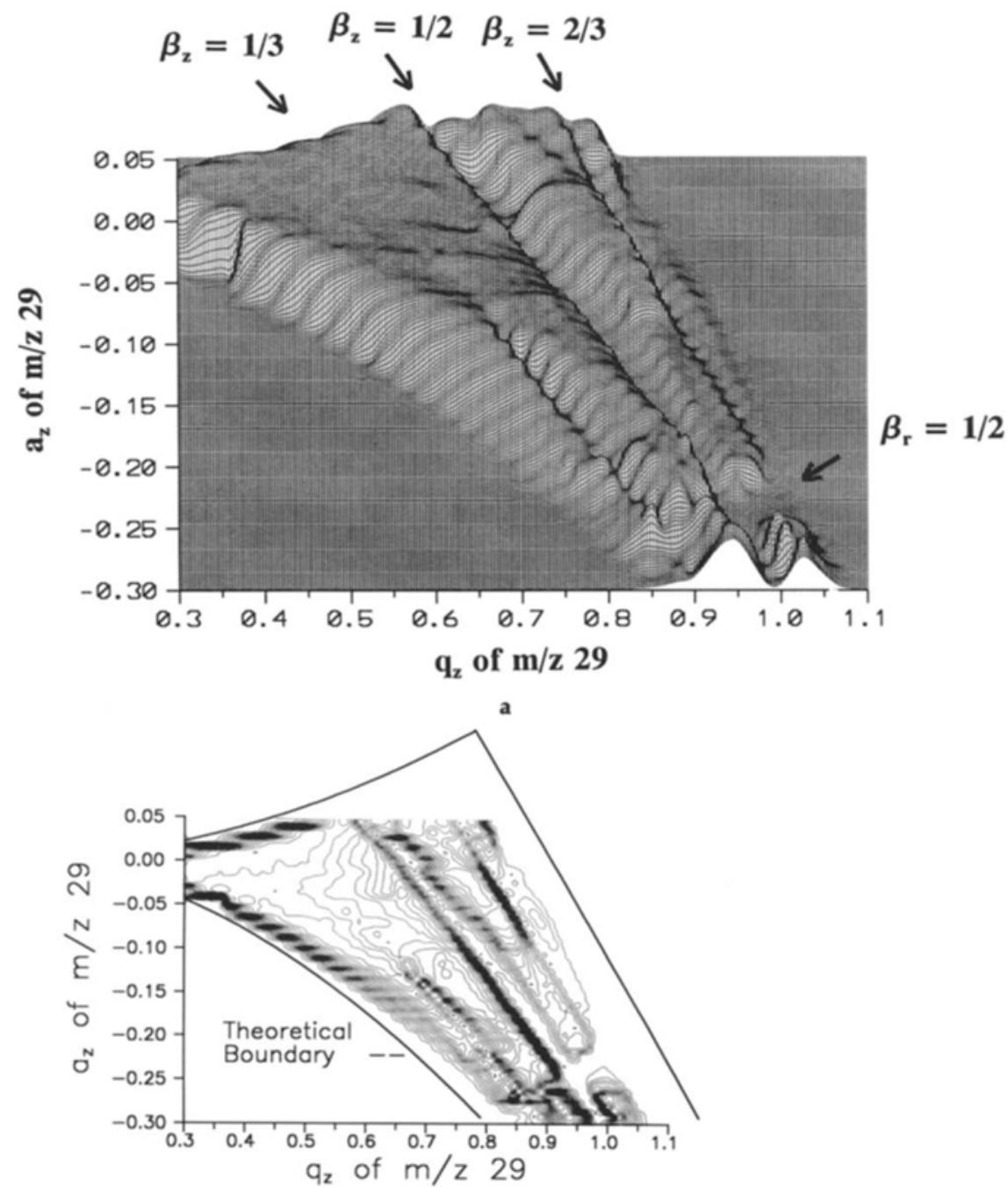

b

Figure 3. (a) Three-dimensional plot and (b) topographical plot of the experimentally determined stability region of mass-selected $\mathrm{m} / z 29\left(\mathrm{C}_{2} \mathrm{H}_{5}^{+}\right)$. Ion intensity following $100 \mathrm{~ms}$ storage is plotted on the $z$-axis, $a_{z}$ is plotted along the $y$-axis, and $q_{z}$ along the $x$-axis. Signal minima fall along the isco- $\beta_{L}$ lines corresponding to $\beta_{z}=1 / 3$ (dodecapolar), $\beta_{z}=1 / 2$ (octopolar), $\beta_{z}=2 / 3$ (hexapolar), and $\beta_{\mathrm{r}}=1 / 2$ (octopolar), respectively.

tions is the uncertainty in the exact $\mathrm{q}_{z}$ of ejection, the value of which is used in eq 15 . The equation assumes that all ions are ejected at $q_{z}=0.906$ due to the axial modulation employed at $530 \mathrm{kHz}$. For low mass-tocharge ratio ions, however, less than $6 \mathrm{~V}_{\mathrm{p}-\mathrm{p}}$ axial modulation can result in ion ejection; as a consequence, 6 $V_{p-p}$ resonant ejection, as employed here, will yield an earlier-than-predicted ejection point and hence a lower $\mathrm{q}_{\mathrm{z}}$ value for resonant ejection during mass analysis. The use of 0.906 in eq 15 rather than the correct (lower) value will yield incorrect (falsely high) calculations for $\mathrm{q}_{\mathrm{z}}$ and $\beta_{\mathrm{z}}$. Replacing 0.906 with the correct $\mathrm{q}_{\mathrm{z}}$ value would improve the agreement between the calculated and theoretical $\beta_{\mathrm{z}}$ values. Other factors, such as uncertainty in the applied DC voltage and subsequent calculation of the $a_{z}$ values, are believed to be minimal since the $D C$ voltages were calibrated with a digital multimeter and the $a_{z}$ values were calculated using the equations for the "stretched" geometry [30].

\section{Parameters Influencing Nonlinear Resanance Effects}

Applied DC voltage. Effects of nonlinear resonances on stored ions appear more pronounced below the $\mathrm{a}_{\mathrm{z}}=0$ line (i.e., with positive DC voltages), as noted in Figure 3. In the lower portion of the stability diagram, increas- 
ingly positive $\mathrm{DC}$ voltages during ion storage of $\mathrm{C}_{2} \mathrm{H}_{5}^{+}$ cause increased ion ejection and CID as well as an increased affected RF range (i.e., width of loss band at $10 \%$ maximum loss) [16]. Figure 4 compares the results of ion storage of $\mathrm{C}_{2} \mathrm{H}_{5}^{+}$using $-5 \mathrm{~V}$ DC $\left(\mathrm{a}_{x}=\right.$ $+0.023)$ to storage using $+10 \mathrm{~V} \mathrm{DC}\left(\mathrm{a}_{\mathrm{z}}=-0.047\right)$. Although not shown in Figure 4, increases in $m / z 27$ and 41 mirror each loss of intensity of $m / z 29$. Note the increased intensity losses and increased "valley" widths with $+D C$. Simulations with the programs ITSIM [28] and HYPERION [29] suggest that applying $+D C$ voltage to the ring electrode causes increased axial and decreased radial amplitudes, while applying $-D C$ to the ring causes increased radial and decreased axial amplitudes. Since the nonlinear resonances abserved in Figure 4 are all of the z-type and thus absorb power only in the z-direction [24], it follows that their effects become more apparent with increased axial motion. Indeed, this agrees with the results by Guidugli et al. [10] in which axial resonant excitation resulted in the observation of nonlinear resonance effects while radial resonant excitation did not.

Also observed while storing $m / z 29$ ions in the

a

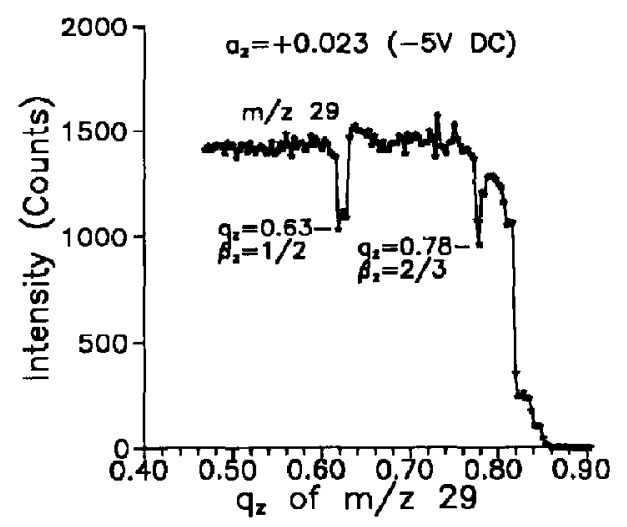

b

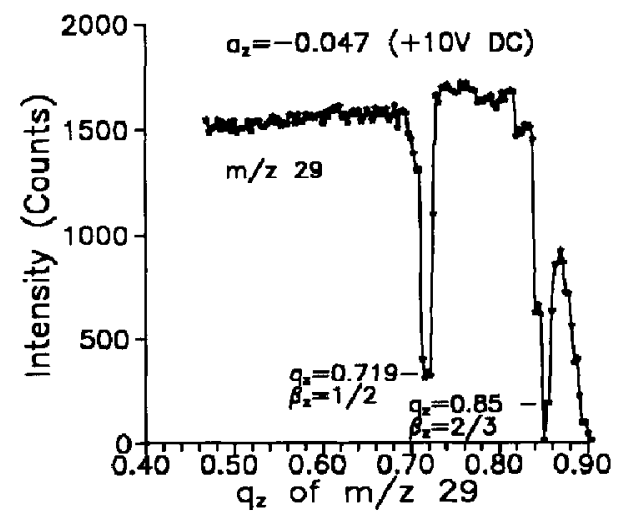

Figure 4. Intensity of mass-selected $m / z 29\left(\mathrm{C}_{2} \mathrm{H}_{5}^{+}\right)$versus $\mathrm{q}_{z}$ of $m / z 29$ following $50 \mathrm{~ms}$ storage at $(\mathrm{a}) \mathrm{a}_{\mathrm{z}}=+0.023(\mathrm{DC}=-5$ V); (b) $\mathrm{a}_{z}=-0.047(\mathrm{DC}=+10 \mathrm{~V})$. lower portion of the stability diagram $\left(\mathrm{a}_{\mathrm{z}}<-0.2\right)$ were further ion-molecule reactions. As stated above, $\mathrm{C}_{2} \mathrm{H}_{5}^{+}$ ions $(m / z 29)$ absorb power and undergo CID to yield $\mathrm{C}_{2} \mathrm{H}_{3}^{+}$ions $(m / z 27)$, which then react with neutral methane to yield $\mathrm{C}_{3} \mathrm{H}_{5}^{+}$ions $(\mathrm{m} / z$ 41). As described below, $\mathrm{C}_{3} \mathrm{H}_{5}^{+}$ions can then undergo $\mathrm{CID}$ to form $\mathrm{C}_{3} \mathrm{H}_{3}^{+}$ions, which then can react with neutral methane to form $\mathrm{C}_{4} \mathrm{H}_{5}^{+}$ions. Indeed, resonance excitation CID of $\mathrm{C}_{3} \mathrm{H}_{5}^{+}$ions at other $\mathrm{q}_{2}$ values confirms this finding.

$$
\begin{aligned}
& \mathrm{C}_{2} \mathrm{H}_{5}^{+} \stackrel{\mathrm{CID}}{{ }^{*}} \mathrm{C}_{2} \mathrm{H}_{3}^{+}+\mathrm{II}_{2} \\
& m / z 29 \quad m / z 27 \\
& \mathrm{C}_{2} \mathrm{H}_{3}^{+}+\mathrm{CH}_{4} \longrightarrow \mathrm{C}_{3} \mathrm{H}_{5}^{+}+\mathrm{H}_{2} \\
& m / z 27 \quad m / z 41 \\
& \mathrm{C}_{3} \mathrm{H}_{5}^{+} \stackrel{\mathrm{CID}}{\rightarrow} \mathrm{C}_{3} \mathrm{H}_{3}^{+}+\mathrm{H}_{2} \\
& m / z 41 \quad m / z 39 \\
& \mathrm{C}_{3} \mathrm{H}_{3}^{+}+\mathrm{CH}_{4} \longrightarrow \mathrm{C}_{4} \mathrm{H}_{5}^{+}+\mathrm{H}_{2} \\
& m / z 39 \quad m / z 53
\end{aligned}
$$

The appearance of the CID product ion, $m / z 39$, from $\mathrm{C}_{3} \mathrm{H}_{5}^{+}$is presumably due to the long storage times near the $\beta_{z}=0$ stability edge for $m / z 41$. The instrumental conditions required to store mass-selected $\mathrm{m} / \mathrm{z}$ 29 near the $\beta_{\mathrm{z}}=1 / 3$ line in the lower portion of the stability diagram (i.e., $-a_{z}$ values), place the $m / z 41$ ion ncar its $\beta_{z}=0$ stability edge. The long storage time used in these experiments (i.e., $100 \mathrm{~ms}$ ) allows the $m / z 41$ ions to absorb power and undergo ejection and CID, as described by the so-called boundary-induced dissociation effect [37].

Ion population. Ion population significantly influences the ability to observe nonlinear resonance effects. Increasing the number of stored ions creates ion-ion repulsions causing displacement of ions from the center of the trap. Since the relative contribution of higher-order fields increases with increasing distance from the center of the trap, displacement from the center will increase ion interaction with nonlinear resonances. The $\mathrm{C}_{2} \mathrm{H}_{5}^{+}$ion $(m / z 29)$ was again used as a chemical probe to investigate the influence of ion population on nonlinear resonance effects. Figure 5a compares nonlinear resonance effects during storage of two ion populations. Ion population is defined here as the intensity of mass-selected $\mathrm{m} / \mathrm{z} 29$ ions (expressed as ion counts) following $50 \mathrm{~ms}$ storage at a $\mathrm{q}_{z}$ value where nonlinear resonances are not observed (i.e., $q_{z}$ $=0.50, a_{z}=0$ ). Although not plotted here, a corresponding intensity increase in $m / z 27$ and 41 mirrors the intensity loss of $m / z 29$. Note the dramatic enhancement of the nonlinear resonance effects with the higher ion population. As observed with the +DC voltage in the previous section, increasing the ion population markedly increases the magnitude of ion loss and affected RF range. Note also that the signal minima shift with increasing ion population. In Figure 

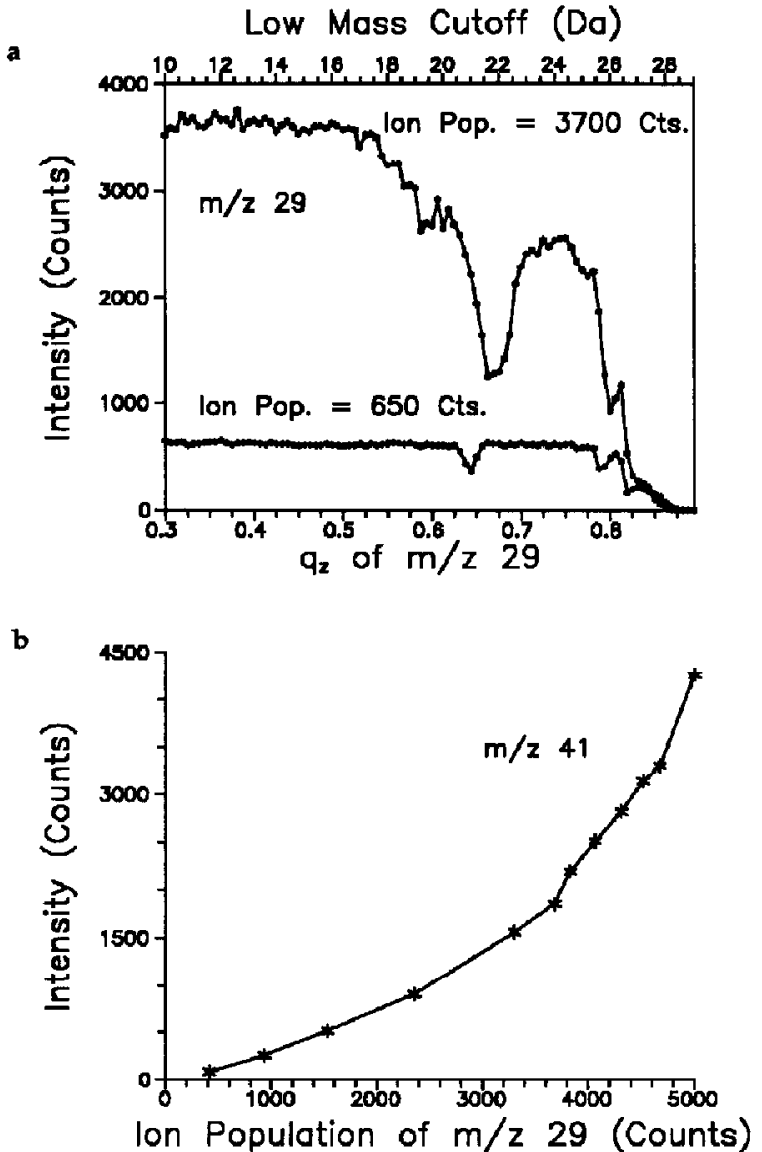

Figure 5. (a) Comparison of ortopolar nonlinear resonance effects for two populations of mass-selected $\mathrm{C}_{2} \mathrm{H}_{5}^{+}$ions $(50 \mathrm{~ms}$ storage); (b) intensity of $m / z 41\left(\mathrm{C}_{3} \mathrm{H}_{5}^{+}\right)$versus ion population of $m / z 29\left(\mathrm{C}_{2} \mathrm{H}_{5}^{+}\right)$in terms of ion count (storage time $=50 \mathrm{~ms}$ ).

$5 a$, the $\beta_{z}=1 / 2$ signal minima occur at $q_{z}=0.656$ and 0.675 for the low and high populations, respectively. An even larger (approximate tenfold) increase in ion population has exhibited a shift corresponding to $\mathrm{q}_{\mathrm{z}}=0.03(\sim 1 \mathrm{Da})$. This effect may be an indication of space-charge-induced resonance shifts [33-35].

Figure $5 \mathrm{~b}$ shows the influence of increasing the ion population during ion storage of $m / z 29\left(\mathrm{C}_{2} \mathrm{H}_{5}^{+}\right)$near the octopolar nonlinear resonance line at $\beta_{z}=1 / 2$ $\left(\mathrm{q}_{\mathrm{z}}=0.65, \mathrm{a}_{\mathrm{z}}=0\right)$. By plotting the intensity of $m / z 41$ $\left(\mathrm{C}_{3} \mathrm{H}_{5}^{+}\right)$as a function of $\mathrm{C}_{2} \mathrm{H}_{5}^{+}$ion population, the extent of $\mathrm{CID}$ (and subsequent ion-molecule reaction to form $m / z 41$ ) can be monitored as the number of $\mathrm{C}_{2} \mathrm{H}_{5}^{+}$ions initially trapped is increased. As more $\mathrm{C}_{2} \mathrm{H}_{5}^{+}$ions are created and trapped, the extent of conversion of $\mathrm{C}_{2} \mathrm{H}_{5}^{+}$to $\mathrm{C}_{3} \mathrm{H}_{5}^{+}$increases. Note that to obtain the data in Figure $5 b$, the $q_{z}$ value for storage was optimized for each ion population by first obtaining data of the type shown in Figure 5a. The exponential trend of Figure $5 \mathrm{~b}$ demonstrates that storage of a very high population of ions markedly increases nonlinear resonance effects. The use of axial modulation during the analytical scan permits a higher ion population to be stored and detected while retaining unit mass resolution [38]. This allows operation of the ion trap with a higher ion population, thus causing more ion-ion repulsions, increased ion displacement from the center of the ion trap, and further interaction between ions and higher-order fields.

Storage time. Storage time directly influences the ability to observe nonlinear resonance effects. Longer storage times allow ions to absorb more power from the higher-order fields, resulting in increased CID or ion ejection. Under the same conditions, ion storage at $\left(q_{z}\right.$, $a_{z}$ ) values where nonlinear resonances are not observed (e.g., $q_{z}=0.5, a_{z}=0$ ) exhibits virtually no ion loss (or CID). Figure 6a compares the influence of storage time of $\mathrm{C}_{2} \mathrm{H}_{5}^{+}$on the observation of the octopolar nonlinear resonance at $\beta_{\mathrm{z}}=1 / 2$. As demonstrated in Figure 6a, longer storage times allow more interaction with the higher-order fields thus causing increased ion losses. Also note that longer storage times cause an earlier onset of ion ejection near the $\beta_{z}=1$ stability edge.
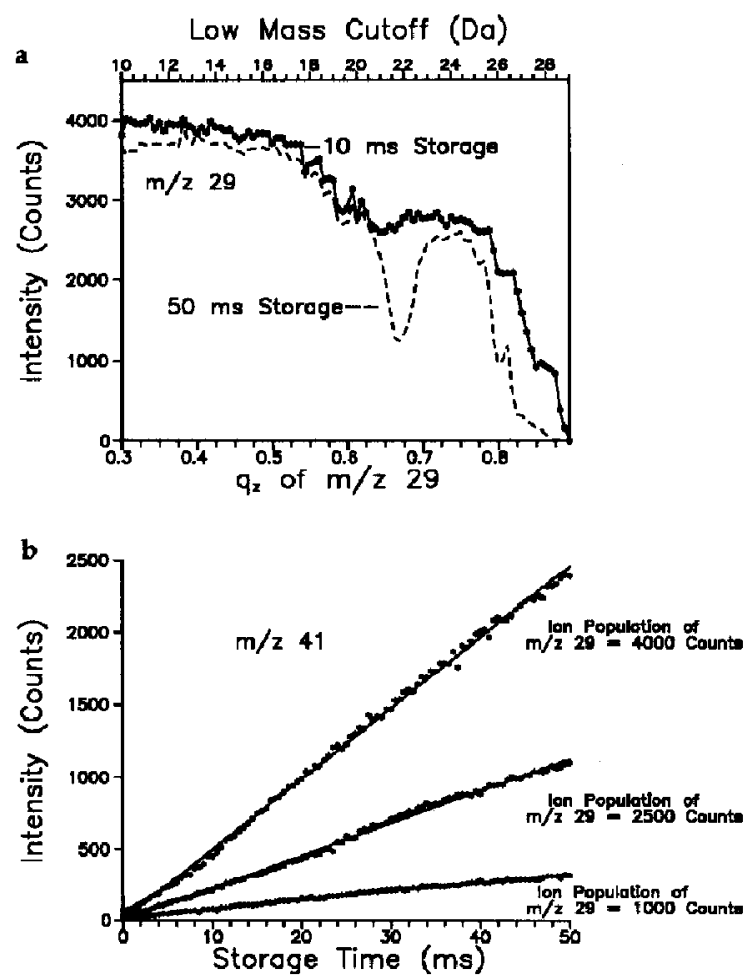

Figure 6. (a) Influence of storage times of mass-selected $\mathrm{C}_{2} \mathrm{H}_{5}^{+}$ ions (population $=4000$ counts) on octopolar nonlinear resonance $\left(\mathrm{a}_{\mathrm{z}}=0\right)$; $(\mathrm{b})$ intensity of $\mathrm{m} / z 41\left(\mathrm{C}_{3} \mathrm{H}_{5}^{+}\right)$versus storage time for three ion populations (i.e., 1000, 2500, and 4000 counts) of mass-selected $\mathrm{C}_{2} \mathrm{H}_{5}^{+}$stored near $\beta_{\mathrm{z}}=1 / 2\left(\mathrm{q}_{\mathrm{z}}=0.65, \mathrm{a}_{\mathrm{z}}=0\right)$. 
Figure $6 \mathrm{~b}$ shows the influence of storage time of three ion populations of mass-selected $\mathrm{C}_{2} \mathrm{H}_{5}^{+}$ions stored at the octopolar nonlinear resonance line near $q_{z}=0.65, a_{z}=0\left(\beta_{z}=1 / 2\right)$. Again, the intensity of $\mathrm{m} / z 41$ is plotted as a gauge for the conversion of $\mathrm{m} / \mathrm{z}$ 29 to its CID product ions. Longer storage times allow the $m / z 29$ ions to absorb more power, causing further CID (and subsequent ion-molecule reactions), as evidenced by the increase in the $\mathrm{C}_{3} \mathrm{H}_{5}^{+}$ion intensity. This behavior has been observed with several ion species at varying ion populations. The linear increase in the $m / z 41$ ion intensity shows that the ability to observe nonlinear resonance effects is markedly increased with longer storage times. As mentioned in the beginning of the Results and Discussion section, the normal EI mode of operation does not employ storage of ions at a given $\left(\mathrm{q}_{7, r} \mathrm{a}_{z}\right)$ point for as Iong as the time shown in Figure $6 \mathrm{~b}$; thus, nonlinear resonances are not as apparent in EI spectra.

Mass-to-charge ratio. Investigations into nonlinear resonance effects of higher mass-to-charge ratio ions were conducted on a series of $n$-alkylbenzenes. Figure $7 \mathrm{a}$ shows the effects of varying the $q_{z}$ value for $50 \mathrm{~ms}$ storage of the $\mathbf{M}^{+}$ion of n-propylbenzene $(m / z 120)$ at $\mathrm{a}_{z}=0(0 \mathrm{~V} \mathrm{DC})$. Near $\mathrm{q}_{2}=0.65$, the ion undergoes ejection and CID to form the product ions at $m / z 91$, 92 , and 105 . The intensity of the product ion $m / z 91$ represents approximately $80 \%$ of all product ions and is therefore the only intensity plotted in Figure $7 a$. Note that the $x$-axis of Figure 7a has been expanded to clearly show some of the fine structure. Although not totally understood, the appearance of a sideband often precedes (at lower $q_{z}$ ) the main loss band. This sideband does not occur during every experiment, but rather seems to be more common when storing a very high ion population. The position of the sideband (in $\mathrm{q}_{z}, \mathrm{a}_{z}$ space) does not correspond to a nonlinear resonance. This may be related to resonance shifts due to space-charge effects [33-35]. Investigations into the origin of the sideband are in progress.

Figure $7 \mathrm{~b}$ shows the effects of varying the $\mathrm{q}_{z}$ value of the $\mathrm{n}$-butylbenzene $\mathrm{M}^{+}$ion ( $m / z$ 134) while storing for $50 \mathrm{~ms}$ in the lower portion of the stability diagram at $\mathrm{a}_{\mathrm{z}}=-0.204(\mathrm{DC}=+200 \mathrm{~V})$. Both the magnitude of ion intensity losses and the affected RF range (i.e., the width of the intensity loss band at $10 \%$ maximum loss) are increased significantly when storing ions in the lower portion of the stability region. Note that the three minima correspond to $\beta_{\mathrm{z}}$ values of $1 / 3$ (dodecapolar, not typically observed at $\mathrm{a}_{\mathrm{z}} \geq 0$ ), 1/2 (octopolar), and $2 / 3$ (hexapolar).

Comparisons of nonlinear resonance effects as a function of mass-to-charge ratio of the n-alkylbenzenes suggest that these effects are virtually independent of mass-to-charge ratio. In other words, ions of all massto-charge ratio values appear to be affected equally, provided an equal number of ions are stored for equal time at $\mathrm{q}_{z}$ values corresponding to nonlinear reso-
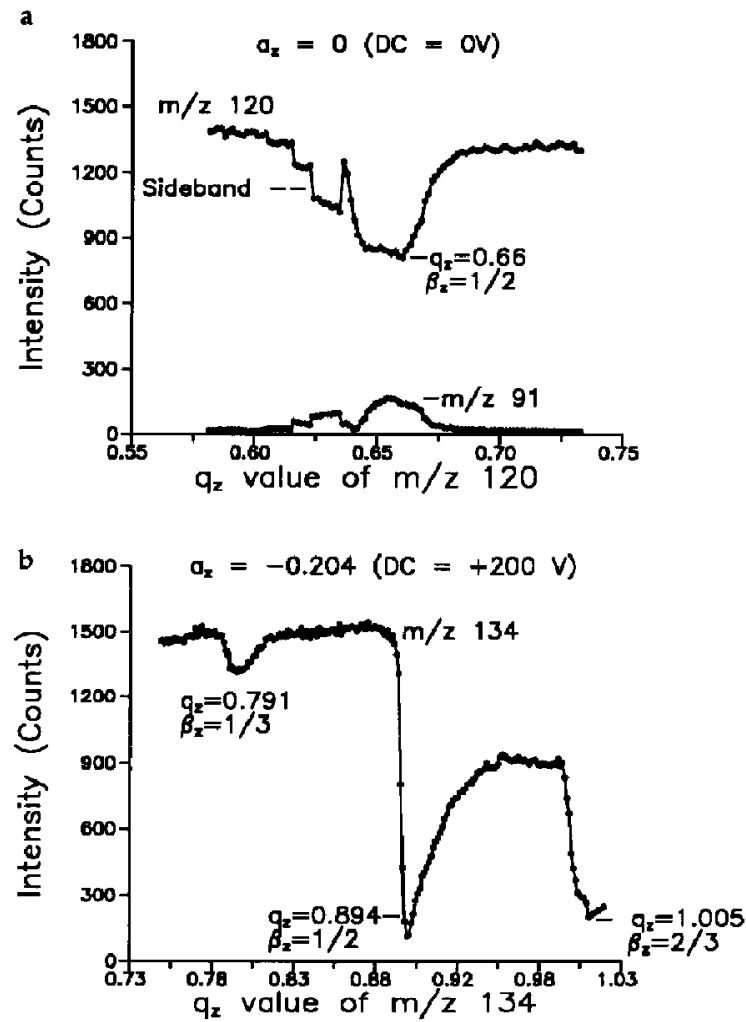

Figure 7. (a) Intensity of mass-selected $m / z 120\left(\mathrm{M}^{+}\right.$of $\mathrm{n}$-propylbenzene) and CID product ion $m / z 91$ versus $\mathrm{q}_{z}$ of $m / z 120$ following 50 ms storage along the $a_{z}=0$ line $(D C=0 \mathrm{~V}$ ); $(b)$ intensity of $m / z 134\left(\mathrm{M}^{+}\right.$. of n-butylbenzene) versus $q_{z}$ following $50 \mathrm{~ms}$ storage along the $\mathrm{a}_{z}=-0.204$ line $(D C=+200 \mathrm{~V})$.

nances. Under identical conditions (number of ions, storage time, $\mathrm{q}_{\mathrm{z}}$ value), ions of differing mass-to-charge ratio exhibit nearly identical intensity losses. The affected RF for each mass-to-charge ratio exhibits a constant range when expressed in terms of $\Delta \mathrm{q}_{z}$. If the affected RF range is plotted in terms of $\Delta m / z$ or $\Delta R F$ voltage, a linear dependence exists due to the inverse relationship of $\mathrm{q}_{\mathrm{z}}$ to mass-to-charge ratio.

\section{Nonlinear Resonance Effects on Cl Spectra}

The significant enhancement of nonlinear resonance effects caused by long storage times and large numbers of stored ions warrants concern when performing CI, particularly when performing mass-selected CI. Operation of the ion trap in the CI mode can employ long ionization and reagent reaction times $(1-20 \mathrm{~ms})$ to accumulate a high population of $\mathrm{CI}$ reagent ions. Additionally, $\mathrm{CI}$ reaction times (reactions between CI reagent ions and analyte neutrals) can approach 100 $\mathrm{ms}$. Under these conditions, nonlinear resonance effects can significantly influence the appearance and intensity of the desired analyte ions. For example, 
mass-selected $\mathrm{CI}$ using $\mathrm{C}_{2} \mathrm{H}_{5}^{+}$of methane at an RF level corresponding to a nonlinear resonance for $m / z$ 29 can result in ionization of the analyte by not only $\mathrm{C}_{2} \mathrm{H}_{5}^{+}(m / z 29)$, but also by $\mathrm{C}_{3} \mathrm{H}_{5}^{+}(m / z 41)$. The conversion of $\mathrm{C}_{2} \mathrm{H}_{5}^{+}$, which favors proton transfer, to $\mathrm{C}_{3} \mathrm{H}_{5}^{+}$, which favors hydride abstraction [39], can alter the resulting $\mathrm{CI}$ mass spectrum.

An example of this is illustrated in Figure 8, where mass-selected reagent ions of methane were reacted with neutral n-butylbenzene. Mass-selected $\mathrm{C}_{2} \mathrm{H}_{5}^{+} \mathrm{CI}$ at an RF level corresponding to a stable region for the reagent ion (e.g., $\mathrm{q}_{\mathrm{z}}=0.50, \mathrm{a}_{\mathrm{z}}=0$; Figure $8 \mathrm{a}$ ) yields the $(\mathrm{M}+\mathrm{H})^{+}$ion of $\mathrm{n}$-butylbenzene $(\mathrm{m} / \mathrm{z} 135)$ due to proton transfer from $\mathrm{C}_{2} \mathrm{H}_{5}^{+}$, as well as a number of fragment ions. Mass-selected $\mathrm{C}_{2} \mathrm{H}_{5}^{+} \mathrm{CI}$ at an RF level corresponding to the octopolar nonlinear resonance for $m / z 29$ at $\beta_{\mathrm{z}}=1 / 2$ (e.g., $\mathrm{q}_{\mathrm{z}}=0.66, \mathrm{a}_{\mathrm{z}}=0$; Figure $8 \mathrm{~b}$ ) results in an increase in the relative ion intensities of several ions, most notably the $(\mathrm{M}-\mathrm{H})^{+}$ion and its associated fragment ions $(\mathrm{m} / z 105$ and 119$)$ as well as the $(M+13)^{+}$adduct ion. These spectra can be compared to the $\mathrm{CI}$ mass spectrum acquired by pure hydride abstraction (shown in Figure 8c), where nbutylbenzene was reacted with mass-selected $\mathrm{C}_{3} \mathrm{H}_{5}^{+}$

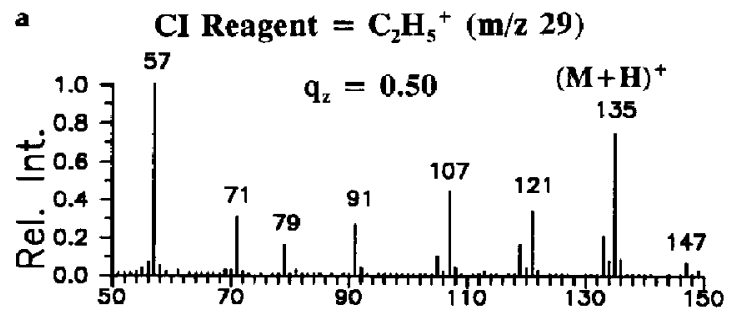

b

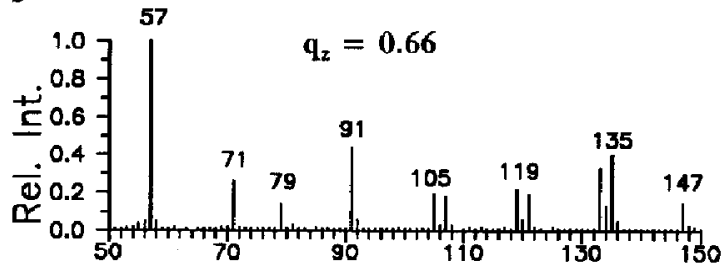

c CI Reagent $=\mathrm{C}_{3} \mathrm{H}_{5}{ }^{+}(\mathrm{m} / \mathrm{z} 41)$

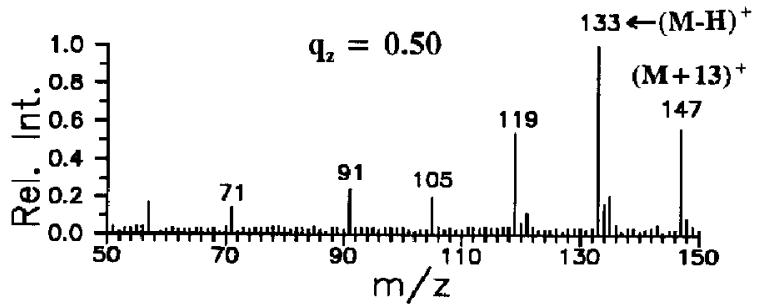

Figure 8. Methane $\mathrm{CI}$ mass spectra of n-butylbenzene acquired by mass-selected $m / z 29\left(\mathrm{C}_{2} \mathrm{H}_{5}^{+}\right) \mathrm{CI}$ at (a) $\mathrm{q}_{2}=0.5$ and (b) $q_{z}=0.66$ for the reagent ion; (c) mass-selected $m / z \quad 41\left(C_{3} \mathrm{H}_{5}^{+}\right)$ CI at $q_{z}=0.5$ for the reagent ion. $(m / z 41)$ at a stable region for the $C I$ reagent ion (e.g., $\left.q_{z}=0.50, a_{z}=0\right)$. The hydride abstraction mass spectrum exhibits a high abundance of the $(\mathrm{M}-\mathrm{H})^{+}$ion and its unique fragment ions, $m / z 105$ and 119 , as well as the $(\mathrm{M}+13)^{+}$ion (presumably arising from the neutral loss of ethylene from the $\mathrm{M}+\mathrm{C}_{3} \mathrm{H}_{5}^{+}$ion). Inspection of Figure $8 \mathrm{~b}$ shows nearly equal contributions of proton transfer and hydride abstraction.

It should be noted that not all compounds will experience such a significant change in CI mechanism based on the identity of the $\mathrm{CI}$ reagent ion, and therefore may not exhibit altered CI spectra. However, acquiring $\mathrm{CI}$ spectra near nonlinear resonances can result in reduced $\mathrm{CI}$ sensitivity due to losses in $\mathrm{CI}$ reagent ions. An example of reduced $\mathrm{CI}$ sensitivity is shown in Figure 9 , in which the intensity of the quantitation ion of an environmental pesticide, $(\mathrm{M} \mid \mathrm{H})^{+}$of carbaryl $(m / z 202)$, is plotted versus the $\mathrm{q}_{\mathrm{z}}$ value of the $\mathrm{CI}$ reagent ion. Figure 9 shows that performing $\mathrm{CI}$ near $q_{z}=0.65$ can result in nearly $50 \%$ loss of ion signal. It becomes apparent that ion trap users must be aware of $\mathrm{q}_{\mathrm{z}}$ values for the $\mathrm{CI}$ reagent ions during acquisition of CI spectra. This is true for both massselected and nonmass-selected CI. For analytes of high molecular weight (i.e., $>400 \mathrm{Da}$ ), $\mathrm{CI}$ is typically performed at high $\mathrm{q}_{\mathrm{z}}$ values of the reagent ion to store the resulting $C I$ product ion at a $\mathrm{q}_{z}$ value which offers efficient storage (i.e., $\mathrm{q}_{\mathrm{z}}>\mathbf{0 . 1}$ ). Particular concern must be made to avoid nonlinear resonances near $q_{z}=0.65$ and 0.8 (for $a_{z}=0$ ) of the $C I$ reagent ion to achieve maximum ion signal for the desired analyte ions. In general, and until the reagent ion population is depleted, increasing the $\mathrm{CI}$ reaction time will increase analyte ion intensity; if storage is performed near a nonlinear resonance line, however, a competing pro-

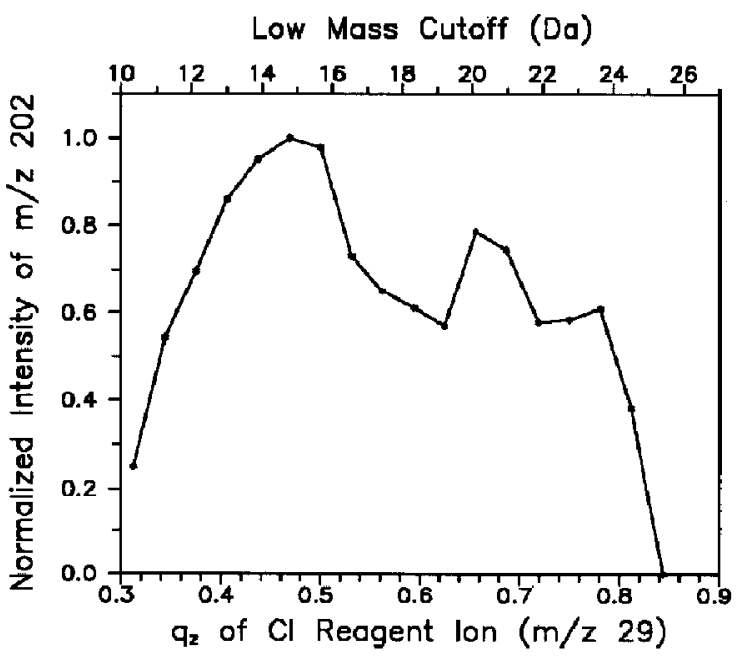

Figure 9. Plot of intensity of the $(\mathrm{M}+\mathrm{H})^{+}$ion of carbary] $(\mathrm{m} / \mathrm{z}$ 202) versus $\mathrm{q}_{2}$ value of the mass-selected $\mathrm{CI}$ reagent ion, $\mathrm{C}_{2} \mathrm{H}_{5}^{+}$ $(m / z 29)$. 
cess will exist between CI reactions to produce analyte ions and $\mathrm{CI}$ reagent ion losses.

\section{Conclusions}

Nonlinear resonance effects on stored ions have been observed in our laboratory with several different mass analyzers on two ITMS systems as well as on a commercial benchtop instrument. The results presented here show that nonlinear resonances can affect virtually any type of experiment performed in the quadrupole ion trap, provided a relatively high population of ions are stored for several milliseconds at RF/DC levels corresponding to a nonlinear resonance condition. Furthermore, any action causing ion displacement from the center of the trap will increase the observed effects due to increased interaction with the higher-order fields near the outer regions. Thus, high ion populations will increase the effects due to ion-ion repulsions which cause ion displacement from the center of the ion trap. Experiments employing long storage times, such as $\mathrm{CI}$, ion-molecule reaction studies, resonance excitation CID, very slow scan rates, and long cool times, can be particularly susceptible to nonlinear resonance effects. Ion trap users should especially be aware of $\mathrm{RF}$ levels during $\mathrm{CI}$, since this mode of operation typically employs high reagent ion populations and long storage times. Furthermore, efficient storage $\left(\mathrm{q}_{z}\right.$ of analyte $\left.>0.1\right)$ of Cl-produced analyte ions of relatively high mass-to-charge ratio typically requires a high $\mathrm{q}_{z}$ for the $C I$ reagent ion $\left(\mathrm{q}_{z}>0.5\right)$ which can place reagent ions near nonlinear resonances.

In general, ion trap users need to be conscinus of RF levels during any experiment to avoid regions of the stability diagram near $\beta_{z}$ values corresponding to nonlinear resonances. The most stable region (free from higher-order nonlinear resonances) can be found in the upper left portion (e.g., $\mathrm{q}_{\mathrm{z}}<0.61, \mathrm{a}_{\mathrm{z}}>-0.05$ ). Nonlinear resonance effects are more significant for ions whose mass-to-charge ratio is less than 1.5 times that of the low mass cutoff, since this dictates $q_{z}$ values greater than 0.61 .

\section{Acknowledgments}

The authors acknowledge the U.S. EPA/EMSL (Cincinnati) for providing funding for this work. They also thank Nathan Yates (University of Florida) for softwarc development and valuable discussions.

\section{References}

1. Stafford Jr., G. C.; Kelley, P. E.; Syka, J. E. P.; Reynolds, W. E.; Todd, J, F. J. Int. J. Mass Spectrom. Ion. Processes 1984, 60, 85.

2. Kelley, P. E.; Stafford Jr., G. C.; Syka, J. E. P.; Reynolds, W. E.; Louris, J. N, Amy, J. W.; Todd, J. F. J. Proceedings of the 33rd Annual ASMS Conference on Mass Spectrometry and Applied Topics; San Diego, CA, May 1985; p 707.
3. Louris, J. N.; Cooks, R, G.; Syka, J. E, P,; Kelley, P. E.; Stafford Jr., G. C.; Todd, J. F. J. Anal. Chem. 1987, 59, 1677.

4. Schwartz, J. C.; Cooks, R. G.; Weber-Grabau, M.; Kelley, P. E. Proceedings of the 36th Annual ASMS Conference on Mass Spectronetry and Allied. Topics; San Francisco, CA, June 1988; $\mathrm{p}$ 634.

5. Louris, J. N.; Amy, J. W.; Ridley, T. Y.; Cooks, R. G. Int. J. Mass Spectrom. Ion Processes 1989, 88, 97.

6. Pedder, R. E.; Yost, R. A.; Weber-Grabau, M. Proceedings of the 37th Annual ASMS Conference on Mass Spectrometry and Allied Topics; Miami Beach, FL, May 1989; p 468.

7. Williams, J. D.; Reiser, H. P.; Kaiser Jr., R. E.; Cooks, R. G. Int. I. Mass Spectrom. Ion Processes 1991, 108, 199.

8. Guidugli, F.; Traldi, P. Rapid Commun. Mass Spectrom. 1991, 5,343 .

9. Morand, K. L.; Lammert, S. A.; Cooks, R. G. Rapid Commun. Mass Spectrom. 1991, 5, 491.

10. Guidugli, F.; Traldi, P.; Franklin, A. M.; Langford, M. L.; Murrell $J_{+}$; Todd, J. F. J. Rapid Commun. Mass Spectrom. 1992, $6,229$.

11. Louris, J. N.; Stafford Jr,, G. C.; Syka, J. E. P.; Taylor, D. Proceedings of the 40th Annual ASMS Conference an Mass Spectrometry and Allied Topics; Washington, DC, May 1992; p 1003.

12. Kaiser, R. E.; Cooks, R. G.; Stafford Jr., G. C.; Syka, J. E. P.; Hemberger, P. H. Int. J. Mass Spectrom. Ion Processes 1991, 106, 79 .

13. Cameron, D.; Hemberger, P. H. Proceedings of the 38th Annual ASMS Conference on Mass Spectrometry and Allied Topics; Tucson, $\mathrm{AZ}$, June 1990; $\mathrm{p} 61$.

14. Todd, J. F. J.; Penman, A. D.; Smith, R. D. int. J. Mass Spectrom. Ion Processes 1991, 106, 117.

15. Griffiths, I. W.; Heesterman, P. J. L. Int. J. Mass Spectrom. Ion Processes 1990, 99, 79.

16. Eades, D. M.; Yost, R. A. Rapid Commun. Mass Spectrom. 1992, 6, 573 .

17. von Busch, F; Paul, W. Z. Physik. 1961, 164, 588.

18. Dawson, P. H.; Whetten, N. R. Int. J. Mass Spectrom. Ion Phys. $1969,2,45$.

19. Dawson, P. H.; Whetten, N. R. Int. J. Mass Spectrom. Ion Phys. $1969,3,1$.

20. Dawson, P. H., Ed. Quadrupole Mass Spectrometry and Its Applications; Elsevier: New York, 1976.

21. March, R. E.; Hughes, R. J. Quadrupole Storage Mass Spectrometry; Wiley: New York, 1989.

22. Beaty, E. C. Phys. Ret. A 1986, 33, 3645.

23. Knight, R. D. Int. I. Mass Spectrom. Ion Phys. 1983, 51, 127.

24. Wang, Y.; Franzen, J.; Wanczek, K. P. Inf. J. Mass Spertrom. Ion Processes 1993, 124, 125.

25. Julian, R. K.; Reiser, H. P.; Cooks, R. G. Proceedings of the 40 th Annual ASMS Conference on Mass Spectrometry and Allied Topics; Washington, DC, May 1992; p 1777.

26. Franzen, J. Int. I. Mass Spectrom. Ion Processes 1991, 106, 63.

27. Yates, N. A.; Griffin, T. P.; Yost, R. A.; Borum, P. R. Proceedings of the 41st Annual ASMS Conference on Mass Spectrometry and Allied Topics; San Francisco, CA, June 1993.

28. Reiser, H. P.; Julian, R. K.; Cooks, R. G. Int. J. Mass Spectrom. Ion Processes 1992, 121, 49.

29. Pedder, R. E.; Yost, R. A. Proceedings of the 36th Annual ASMS Conference on Mass Spectrometry and Allied Topics; San Francisco, CA, June 1988; p 632.

30. Johnson, J. V.; Pedder, R. A.; Yost, R. A. Rapid Commun. Mass Spectrom. 1992, 6, 760 .

31. Weber-Grabau, M.; Kelley, P. E.; Syka, J. E. P.; Bradshaw, S. C.; Brodbelt, J. S. Proceedings of the 35th Annual ASMS 
Conference on Mass Spectrometry and Applied Topics; Denver, CO, May 1987; p 1114.

32. Harrison, A. G. Chemical lonization Mass Spectrometry; CRC: Boca Raton, FL, 1983.

33. Fischer, E. Z. Physik. 1959, 156, 1.

34. Sheretov, E. P.; Zenkin, V. A.; Samodurov, V. F. Sor. Phys. Tech. Phys. 1973, 18, 282.

35. Fulford, J. E.; Hoa, D.-N.; Hughes, R. J.; March, R. E.; Bonner, R. F.; Wong, G. J. J. Vac. Sci. Technol. 1980, 17, 829.

36. Wang, Y.; Franzen, J. Proceedings of the 12th Intertational Mass
Spectrometry Conference; Amsterdam, The Netherlands, August 1991.

37. Paradisi, C.; Todd, J. F. J.; Traldi, P.; Vettori, U. Org. Mass Spectrom. 1992, 27, 251.

38. Tucker, D. B.; Hameister, C. H.; Bradshaw, S. C.: Hoekman, D. J-; Weber-Grahau, M. Proceedings of the 36th Annual ASMS Conference on Mass Spectrometry and Allied Topics; San Francisco, CA, June 1988; p 628.

39. Berberich, D. W.; Hail, M. E.; Johnson, J. V.; Yost, R. A. Int. J. Mass Spectrom. Ion Processes 1989, 94, 115. 